mismatch between substrate and overgrowth ${ }^{4}$.

The orientation relationship that we report results from a correlation of $d$-spacings from electron diffraction, and the more accurate $d$-spacings from X-ray diffraction; such a correlation cannot be obtained if the orientation relationship proposed by Mackay ${ }^{1}$ is assumed. Nor is there evidence to postulate a relaxation in the Laue condition, since scanning electron microscopy (see Fig. 3, ref. 3) indicates that the green rust crystals are $\sim 0.1 \mu \mathrm{m}$ in thickness or greater. The elongation of diffraction spots is usually associated with microcrystals which have critical dimensions of much less than 0.1 $\mu \mathrm{m}$. Electron diffraction suggests that the green rust platelets are well-crystallised. An inspection of the rhombohedral unit cell shows that a flat crystal with a $\overline{(5} \overline{6} \overline{6})$ habit plane may have hexagonal geometry.

Turning to the point raised by Brindley and $\mathrm{Bish}^{5}$ : in our experiments green rust is grown in closed vessels by the corrosion of cast iron in solutions which are deoxygenated by purging with nitrogen. Thus the level of dissolved carbon dioxide is very low in non-carbonate solutions. For example, green rust and magnetite were formed by the corrosion of cast iron at $50^{\circ} \mathrm{C}$ in distilled, deionised water containing 0.4 p.p.m. of oxygen. Chemical analysis of the water gave the following results: before corrosion, $p \mathrm{H}=7.4$, alkalinity $=5.0$ p.p.m. ; after corrosion, $p \mathrm{H}=$ 6.6 , alkalinity $=5.5$ p.p.m.; alkalinity $=$ $\left[\mathrm{CO}_{3}{ }^{2-}\right]+\left[\mathrm{HCO}_{3}{ }^{-}\right]+\left[\mathrm{CO}_{2}\right]+\left[\mathrm{OH}^{-}\right]$. Since, for $\mathrm{H}_{2} \mathrm{CO}_{3}$ at $50{ }^{\circ} \mathrm{C}, K_{1}=5.19 \times$ $10^{-7}$ and $K_{2}=6.73 \times 10^{-11}$ (ref. 6) the bicarbonate ion is the predominant species in this $p \mathrm{H}$ range, and the carbonate ion concentration is extremely low.

In another experiment, green rust produced in sodium carbonate solution, $\left[\mathrm{CO}_{3}{ }^{2-}\right]=200$ p.p.m. was examined using infrared spectroscopy. The presence of the hydroxyl group was clearly shown but the carbonate ion was not detected. In view of the comments of Brindley and $\mathrm{Bish}^{5}$, a more extensive infrared spectroscopic investigation is being undertaken, but at present, our experiments do not support the view that the presence of the carbonate ion is particularly significant in the formation of green rusts by the corrosion of cast iron.

These and other points relating to processes involved in corrosion of cast iron will be developed elsewhere.

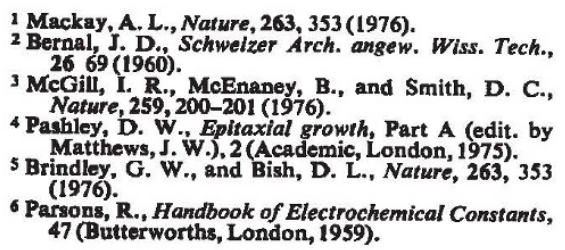

\section{Production of heavy elements in neutron stars}

Chechetkin and Kowalski ${ }^{1}$ have proposed that the production of heavy elements in nature occurs by the ejection of matter from a neutron star surface. Their suggestion relies on the results of Chechetkin and Bisnovatyi-Kogan ${ }^{2,3}$, who find such matter to be composed before ejection of 'jumbo' nuclei $(Z \gtrsim 160$; $A \gtrsim 640$ ). These results were derived using a highly uncertain extrapolation of binding energies into the extreme neutronrich region and in the absence of fission. Their justification of the neglect of fission is based on a necessarily very crude estimate of fission lifetimes.

The nuclear stability against spontaneous fission is the result of a barrier in the potential energy curve of a nucleus as a function of deformation. In the framework of the macroscopic-microscopic method ${ }^{4,5}$, the potential energy can be divided into two parts: (1) a macroscopic contribution that takes into account the smooth variation of the energy as a function of nucleon number and deformation, and (2) a microscopic contribution that includes shell effects which are significant only near the magic numbers for neutrons or protons. The macroscopic energy is calculated using the liquid-drop or droplet model of the nucleus, whereas the microscopic contribution is evaluated from the energy spectrum associated with an appropriate potential well.

As the proton number and the neutron excess of the nucleus are increased, the stability against fission resulting from the macroscopic energy is reduced both by the increased repulsion of the Coulomb force with the addition of protons and by the decreased surface tension of the nucleus with the addition of neutrons. It is the rapid decrease of the macroscopic energy as a function of deformation that causes nuclei beyond the actinide region ( $A \gtrsim 260$ ) to be unstable with respect to spontaneous fission. Beyond the actinide region, only strong single-particle effects near neutron or proton closed shells (as for the case of the superheavy island $A \simeq 298, N \simeq 184, Z \simeq 114$ ) can offer stability against spontaneous fission. In the immediate region surrounding the superheavy island, the fission barriers of nuclei are virtually non-existent ${ }^{6}$.

Furthermore, in a neutron capture the neutron separation energy in the compound nucleus plus the kinetic energy of the incoming neutron easily lead to subsequent fission. Consequently, any process of successive neutron captures will be terminated by neutron-induced fission before significant spontaneous fission can occur. Recent calculation $s^{6,7}$ of fission barrier heights and neutron separation energies in the $r$-process region indicate that the astrophysical $r$ process may well be terminated by neutroninduced fission in the mass region $A \sim 280$. It should, however, be pointed out that the exact point of this termination is very uncertain because of an extrapolation of surface-asymmetry and closed-shell effects from the region of known nuclei and because of the use of the Strutinsky procedure. The termination point may be somewhat higher $(A \sim 350)$, consistent with the possible discovery of superheavy elements in pleochroic halo inclusions $^{8}$ and their production in a conventional $r$ process ${ }^{9}$. In spite of the uncertainties of the calculations of fission barriers $^{6,7}$, we believe that the existence of nuclei as large as $A=640, N=480$, $Z=160$ is definitely ruled out. We should also mention that similarly large nuclei had been predicted in equilibrium conditions in neutron star matter ${ }^{10,11}$. This, however, has also been conclusively shown to be incorrect ${ }^{12-14}$. In conclusion, the nuclear basis of Chechetkin and Kowalski's suggestion for the production of superheavy elements is unfounded although it may well be that these elements can be produced in an $r$ process occurring in the surface material of neutron stars. Studies along these lines are under way.

This work was supported in part by the NSF. We acknowledge the hospitality of the Aspen Center for Physics.

\section{J.- ROBERT BUCHLER}

Department of Physics and Astronomy, University of Florida,

Gainesville, Florida 32611

William A. Fowler Michael J. Newman

W. K. Kellogg Radiation Laboratory, California Institute of Technology, Pasadena, California 91125

\section{Michael Howard}

Department of Physics, Northwestern University, Evanston, Illinois 60201

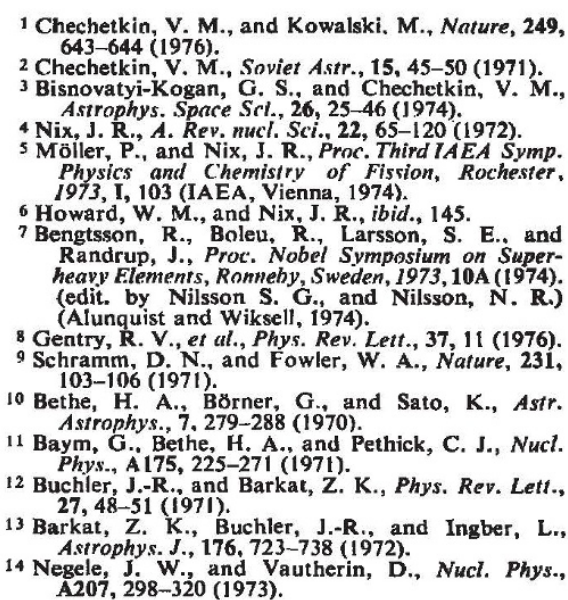

